\title{
Traduire
}

Une autre perspective sur r tr traduction

Revue française de la traduction

$242 \mid 2020$

Passons au vert

\section{La transition énergétique : un enjeu de formation}

\section{Muryel Derlon et Martina Mayer}

\section{(2) OpenEdition}

\section{Journals}

Édition électronique

URL : http://journals.openedition.org/traduire/2033

DOI : 10.4000/traduire.2033

ISSN : 2272-9992

\section{Éditeur}

Société française des traducteurs

\section{Édition imprimée}

Date de publication : 30 juin 2020

Pagination : 102-112

ISSN : 0395-773X

\section{Référence électronique}

Muryel Derlon et Martina Mayer, «La transition énergétique : un enjeu de formation », Traduire [En ligne], 242 | 2020, mis en ligne le 15 juillet 2020, consulté le 21 décembre 2020. URL : http:// journals.openedition.org/traduire/2033 ; DOI : https://doi.org/10.4000/traduire.2033 


\section{La transition énergétique: un enjeu de formation}

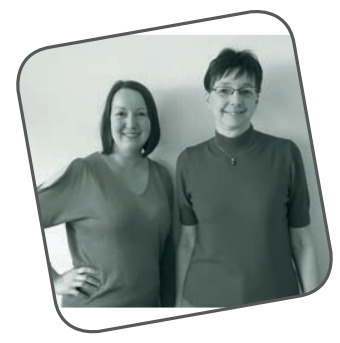

Muryel Derlon, Martina Mayer

La transition énergétique, concept-clé dans la lutte contre la crise climatique, est devenue l'un des grands sujets de notre époque. Dans ce contexte, exclure le développement durable de la formation universitaire de futurs traducteurs paraît impensable aux auteures de cette contribution.

Dans notre fonction d'enseignantes-chercheuses au sein de I'Institut de traductologie (INTRAWI) de l'université d'Innsbruck, nous avons donc élaboré un programme pour deux cours de traduction, thème et version, qui appartiennent à la formation master de traductologie, option traduction spécialisée. Les deux cours se déroulent sur quinze semaines et chaque séance hebdomadaire dure 90 minutes. Cette unité d'enseignement, intitulée Fachübersetzen - Technik: die Energiewende (en français: Traduction spécialisée - technique: la transition énergétique), permet aux futurs diplômés non seulement d'acquérir une vaste connaissance générale autour des enjeux que nous impose le réchauffement 
climatique mais encore, et surtout, de se préparer à l'activité traduisante dans le domaine des énergies renouvelables.

Ainsi, la formation que nous proposons répond premièrement à une responsabilité vis-à-vis des étudiants, que nous devons préparer au mieux à leur futur rôle de "passeurs» linguistiques et culturels, et deuxièmement à une responsabilité vis-à-vis de la société dont chaque membre devrait être sensibilisé à la problématique de la crise climatique.

En exposant en détail le concept de ces deux cours complémentaires, nous espérons pouvoir inspirer d'autres établissements de formation, ainsi que leurs enseignants qui, eux aussi, sont en quête permanente d'une dynamisation de leurs approches didactiques. Esquissons donc brièvement le contexte de formation mis en place à Innsbruck, lequel détermine la conception générale de notre programme d'études et l'orientation de chacun de nos cours.

\section{Contexte de formation}

\section{Contexte général}

Dans l'espace germanophone, les formations proposées aux futurs traducteurs mettent un accent particulier sur une profonde réflexion théorique, associée à des activités pratiques, car la traduction dite «appliquée» ne suffit plus pour permettre aux jeunes diplômés de proposer avec succès leurs services sur le marché du travail. Cette méthode de travail leur permet de mettre à profit les théories acquises pour fournir d'excellentes prestations de service d'une part et de faire évolver la traductologie, domaine académique en plein essor, en contribuant activement à la recherche, d'autre part.

En Autriche, l'enseignement de la traductologie/traduction est confié aux universités (instituts de traductologie de Vienne, Graz et Innsbruck). Leurs fonctions sont clairement définies dans la législation universitaire en vigueur: Universitätsgesetz 2002 UG. Cette loi prévoit que les universités sont chargées d'assurer l'évolution et l'enseignement de la recherche et du discours académique dans leurs domaines respectifs et de préparer en parallèle les futurs diplômés à une activité professionnelle (voir Rechtsinformationssystem des Bundes, 2020, spécifiquement paragraphes 1 et 3 ). 
Par conséquent, même nos cours à caractère plutôt pratique sont soumis à cette directive qui prévoit d'ancrer la réflexion scientifique dans chaque unité d'enseignement.

Après cette première mise au point, la question se pose de savoir à quel stade de la formation il convient d'introduire cette professionnalisation alliant théorie et pratique. La licence n'est-elle qu'une propédeutique de la traductologie et le master qu'une simple première étape de la professionnalisation, qui se consolidera ensuite par l'entrée dans la vie active? Ou souhaite-t-on placer la préparation à une carrière professionnelle et l'introduction à la pratique scientifique davantage en amont, c'est-à-dire dès la licence, et ainsi obtenir avec le master un véritable niveau de perfectionnement?

\section{Contexte à I'INTRAWI}

L'INTRAWI est dans ce domaine un véritable pionnier en la matière, car notre institut propose un cursus intitulé Bachelorstudium Translationswissenschaft (licence en traductologie), qui permet aux étudiants d'acquérir une première qualification professionnelle en trois langues (leur langue maternelle, ainsi que deux langues étrangères) grâce à une offre variée de cours pour un total de 180 ECTS. Selon le programme d'études, les licenciés sont qualifiés pour traduire, sur le marché, des textes simples à caractère général (voir université d'Innsbruck, 2016, p. 1, 2 et 12).

Le master comptant 120 ECTS doit par conséquent conduire les étudiants à une maîtrise complète de leur métier et leur assurer une compétitivité absolve sur un marché qui n'est pas protégé par les pouvoirs publics. Le cursus reflète clairement ces objectifs. Dans l'option traduction spécialisée, les étudiants sont amenés à traduire des textes hautement spécialisés issus de différents domaines, tout en utilisant les technologies modernes, en appliquant les méthodes de la terminologie et en maîtrisant les stratégies qui leur permettront de s'adapter rapidement et efficacement à d'autres domaines de traduction. Les deux autres filières d'études proposées à Innsbruck, traduction littéraire et audiovisuelle ainsi qu'interprétation de conférences, visent bien entendu un niveau de perfection équivalent (voir université d'Innsbruck, 2019, p. 1 et 2). 
Dans le cadre du master en traduction spécialisée, les cours de traduction proposent, entre autres, les domaines de spécialisation classiques tels que le droit, l'économie, la médecine ou la technique. Les enseignants dispensant ces cours ont une certaine latitude pour orienter le contenu principal de leurs enseignements.

\section{Contexte des cours de traduction spécialisée}

En tant que responsables des deux cours de traduction mentionnés plus haut, nous avons choisi la thématique de la transition énergétique. Une fois ce choix fixé, nous avons créé une unité d'enseignement incluant des cours de thème et version. Les étudiants y ont abordé la transition énergétique en général, les objectifs climatiques européens, français et autrichiens, les lois et l'ensemble des mesures concernées et, plus particulièrement, l'hydroélectricité et le photovoltaïque comme exemples d'énergies vertes. Les avantages de la thématique envisagée sont évidents:

- sur le plan idéologique, en participant à ces cours, les étudiants auront découvert un domaine qui leur permettra d'assumer une responsabilité sociale en tant qu'acteurs de la médiation linguistique et de participer activement à la construction du monde dans lequel nous vivons;

- sur le plan économique, les étudiants auront été initiés à un domaine de travail très vaste dont l'actualité n'est plus à prouver. Ce sujet restera encore longtemps actuel dans les affaires mondiales et offrira donc des opportunités de travail non négligeables;

- sur le plan pédagogique, afin de motiver nos étudiants à suivre volontairement plusieurs fois les cours (chose possible en Autriche, car les étudiants peuvent suivre des cours déjà validés en tant qu'auditeurs libres), nous avons diversifié leurs contenus sur plusieurs cycles;

- sur le plan de la didactique de la traduction, le sujet choisi nous a permis de confronter les étudiants à une très grande diversité de genres textuels, de conventions 
qui en découlent, et de langues de spécialité ${ }^{1}$, ce qui est essentiel pour les préparer aux multiples attentes du marché.

Afin de mettre tous ces aspects en œuvre avec la plus grande efficacité pédagogique possible, nous nous sommes appuyées sur une combinaison de pratiques traditionnelles, qui ont déjà fait leurs preuves, associée à un large éventail d'approches didactiques innovantes.

\section{Concept de cours}

\section{Enseignement traditionnel}

Pour l'enseignement traditionnel, la combinaison de diverses activités s'est avérée fort efficace: grâce à l'alternance d'analyse de texte, de traduction, d'autorévision et de révision externe, non seulement ladite "compétence de transfert» a pu être affinée, mais les compétences méthodologiques et stratégiques, essentielles tant en amont qu'en aval du processus de traduction, ont aussi été améliorées. Pensons par exemple à l'estimation correcte du délai et du prix d'une traduction professionnelle, à l'autorévision ou au recours à une révision externe, donc à des mesures qu'un traducteur doit savoir employer pour garantir l'excellente qualité de son travail.

Toujours dans le cadre de cet enseignement traditionnel, nous avons demandé à nos étudiants d'élaborer, tout au long du semestre un «glossaire de traducteur»; il recense les termes les plus difficiles, est évolutif et disponible pour de futures traductions.

Par ailleurs, nous avons veillé à utiliser des modes de transmission variés: présentations individuelles des étudiants avec feed-back du groupe, discussions libres ou dirigées en séance plénière, exposés et échanges entre les participants sur les différents acquis. Ces modes de présentation ont activé à plusieurs niveaux les compétences des étudiants

1. Nous utilisons "langues de spécialité» au pluriel parce que le domaine de spécialité de la transition énergétique se décline en sous-domaines qui disposent chacun d'une terminologie propre. En cours, cet aspect permet aux étudiants d'acquérir des stratégies d'adaptation rapides à différents sujets. 
en communication et en argumentation et les ont ainsi préparés à différents contextes de discussion: par exemple, ils étaient mieux armés pour argumenter au sein d'une équipe sur de possibles stratégies de traduction ou pour justifier avec conviction un choix de traduction face à un client.

En outre, nous avons eu recours à plusieurs métho des de travail: travail individuel, en binôme, en équipe, tâches à effectuer en présence de l'enseignante avec correction immédiate et, inversement, observation de cette dernière par les étudiants lors de la recherche et de l'exploitation de ressources documentaires ciblées. L'ensemble de ces activités a encouragé les étudiants à être plus innovants dans la réalisation de leurs traductions et leur a permis d'identifier et de corriger plus rapidement les erreurs dans leur propre travail.

\section{Enseignement innovant}

Mais la pièce maîtresse de notre concept de cours est sans aucun doute la création d'un cadre d'enseignement innovant. Pour ce faire, nous avons intégré dans nos cours des partenaires externes. Nous avons réussi à persuader les Innsbrucker Kommunalbetriebe (IKB), les services communaux d'Innsbruck et principal acteur énergétique de la ville, à nous soutenir dans notre tâche; et avec la Tiroler Wasserkraft AG (TIWAG), principal fournisseur d'électricité au Tyrol, nous sommes parvenues à rallier une autre entreprise clé de l'industrie énergétique régionale à notre cause. Nous tenons à remercier sincèrement ces deux entreprises pour le soutien apporté, car elles ont largement favorisé le processus d'apprentissage des étudiants en enrichissant nos cours grâce à des conférences et des excursions.

Ainsi les étudiants ont-ils pu quitter leur salle de cours douillette pour visiter un parc photovoltaïque, découvrir une centrale électrique d'eau potable ou encore apprendre dans une station d'épuration comment le traitement des boues peut produire de l'énergie. Ces excursions sont précieuses non seulement parce qu'elles rendent concrètes et bien visibles les informations dispensées en cours, mais aussi parce qu'elles confrontent les participants à un nouveau type de communication spécialisée. En effet, les salariés de ces entreprises partenaires, formés pour transmettre 
des connaissances spécialisées à un public de profanes, ont permis aux étudiants de participer à une communication technique verticale à fort caractère de vulgarisation. Ils ont ainsi pu acquérir une métacompétence importante, puisque les traducteurs ne cessent d'être amenés, dans leur travail quotidien, à adapter les textes à un groupe cible et à évaluer quelles connaissances peuvent ou non être présupposées chez les destinataires du texte cible.

Nous avons également pu accueillir des experts au sein de notre institut. Mandatés par les entreprises partenaires, ils sont intervenus en cours dans leur domaine de spécialisation tel que le fonctionnement des centrales hydroélectriques et de leurs composants, le phénomène du coup de bélier dans les conduites forcées ou les implications de la législation dans la protection de l'environnement lors de la mise en place d'installations de production d'énergie verte. Contrairement à la communication verticale mentionnée ci-dessus, les étudiants ont acquis pendant ces conférences des connaissances hautement spécialisées comme s'ils participaient à une communication technique horizontale, même si, pour favoriser la compréhension de ces données très spécialisées, ces intervenants ont fait preuve de pédagogie. Dans ce contexte d'apprentissage, les étudiants devaient comprendre globalement les informations techniques, les comparer en détail avec les contenus de leur propre traduction, poser les bonnes questions de compréhension et en même temps, si possible, s'approprier le «jargon» technique des orateurs pour l'utiliser à bon escient dans leur travail. L'apprentissage de ces mécanismes est absolument essentiel pour créer une communication efficace avec les clients.

Finalement, nous avons conclu un partenariat fructueux avec le Département d'ingénierie hydraulique de l'Institut pour l'infrastructure de l'université d'Innsbruck. Les collègues de cet institut avaient besoin de la traduction allemande d'un manuel de plus de cent pages qui devait permettre à leurs étudiants d'utiliser un logiciel francophone de modélisation des conduites forcées pour les centrales hydrauliques. Les futurs traducteurs de nos cours de master ont ainsi eu la possibilité de faire un travail réellement commandité par un client dans le cadre d'un projet de traduction universitaire non lucratif. Nous avions déjà par le passé souvent réalisé 
de telles coopérations et avons donc pu nous appuyer sur les retours positifs et notre expérience acquise à cet égard. Les ouvrages de référence ayant comme sujet les "projets de traduction» témoignent également, sans équivoque, de leur utilité dans l'apprentissage du métier (voir par exemple Krenzler-Behm, 2013 ou Mayer, 2017). Mais dans ce cas précis, ce travail s'est déroulé dans un cadre très inhabituel. En effet, les étudiants du Département d'ingénierie hydraulique, destinataires du texte cible, ont collaboré étroitement avec ceux de notre institut pour leur fournir le soutien technique nécessaire dont tout traducteur, conscient de ses limites, a absolument besoin pour comprendre et reproduire correctement des contenus d'une telle complexité. L'objectif ici était double: d'une part, les futurs ingénieurs, tous en première année de licence, devaient apprendre à communiquer avec des non-spécialistes et pouvaient en même temps consolider leurs connaissances techniques en les transmettant à l'équipe de traduction; d'autre part, nos étudiants ont pu non seulement apprendre à gérer la relation client, mais aussi à cerner les dangers d'une communication technique émanant d'un personnel semi-professionnel dont on ne peut supposer avec certitude que les informations ou les explications fournies soient complètes et absolument correctes.

Ce point a été particulièrement utile pour notre concept de cours, car dans cette coopération, la communication entre le traducteur et son client reflétait bien plus la réalité du quotidien que ne l'avait fait le dialogue avec les experts rencontrés en cours. En général, dans le monde professionnel, les clients sont bien moins sensibilisés au besoin d'informations du traducteur qu'un conférencier invité. Ce constat oblige le traducteur à devoir identifier ses propres lacunes malgré l'opacité du sujet due à l'ignorance du domaine de spécialité et à se procurer les informations manquantes auprès du client, même si celui-ci n'en voit pas la nécessité.

L'expérience menée ici a été très convaincante. D'un côté, les étudiants en génie hydraulique se sont rendu compte à quel point il était difficile de mettre en mots leur propre expertise et ont décrit la collaboration à ce niveau à la fois comme un défi et un enrichissement; de l'autre, nos étudiants en master ont noté à quel point il est nécessaire de faire ses propres recherches terminologiques et documentaires en 
complément des explications données par le client, mais en même temps aussi combien il peut être difficile de se motiver encore et toujours pour relancer la communication ciblée avec ce dernier afin de l'obliger à une coopération performante.

Dans notre concept de cours englobant jusqu'ici des compétences traductives, linguistiques, personnelles et interrelationnelles, il nous a semblé essentiel d'accorder une attention toute particulière à la traductique, puisque les technologies modernes de la traduction font désormais partie intégrante de la vie quotidienne du traducteur. Or, en ce qui concerne la TAO, le cursus actuellement en place à l'INTRAWI prévoit des cours spécifiques qui préparent nos étudiants à l'utilisation efficace de différents logiciels. Nous avons donc décidé de compléter ces acquis dans nos cours en montrant aux étudiants comment utiliser intelligemment la traduction automatique dans le domaine de la transition énergétique. Tout d'abord, ils ont été répartis en équipes, puis chacune d'entre elles a reçu trois textes à traduire vers sa langue maternelle. Ces textes traitaient tous de la transition énergétique, mais appartenaient à des types et à des genres différents. Les équipes ont travaillé successivement sur ces textes, chacune avec un logiciel informatique spécifique. Elles avaient pour tâche d'identifier les erreurs de traduction faites par ce logiciel, ainsi que la terminologie technique, les noms propres, les pronoms, la syntaxe, etc. à l'origine de ces erreurs.

Après une introduction théorique sur la question, doublée de conseils pratiques, les étudiants ont franchi ces différentes étapes de manière autonome, mais en recevant le retour et les commentaires des deux enseignantes. Ils ont ainsi découvert le potentiel et les limites de la TA dans différents cas de figure, eu un aperçu de l'importance de la pré-édition et commencé à automatiser les diverses étapes d'une post-édition professionnelle. À l'issue de cette échappée technologique, les étudiants ont été invités à présenter à leurs condisciples les conclusions du travail de leur équipe. Lors d'une présentation d'une heure, ils ont exposé le fonctionnement des logiciels utilisés, ainsi que les implications de leur usage sur le plan déontologique. Ainsi ont-ils pu non seulement partager avec les autres étudiants leurs connaissances théoriques sur les outils respectifs, mais surtout 
les approfondir dans le cadre de travaux de recherche à orientation scientifique et éthique.

\section{Conclusion et perspectives}

Après avoir achevé deux cycles de cours sur la transition énergétique, nous avons obtenu des résultats très satisfaisants. Dans les évaluations anonymes auxquelles nos collègues et nous-mêmes sommes régulièrement soumis, les étudiants ont déclaré qu'ils trouvaient très utiles la combinaison des différentes méthodes, ainsi que le développement des multiples compétences qui en résulte. Le caractère dynamique des cours répond à leur besoin de quitter en douceur et progressivement l'environnement protégé du cadre de formation et leur permet de se familiariser avec leurs futures responsabilités envers le client. L'initiation à la TA a suscité des réactions très positives de leur part, car ils manquent souvent d'occasions pour apprendre à connaître les différents outils informatiques en détail. De plus, pouvoir analyser et étudier toute une série de textes leur donne confiance en leurs capacités dans l'usage de la traductique. Enfin, le choix du thème, la transition énergétique, a séduit les étudiants qui appartiennent à une génération de plus en plus préoccupée par le destin de notre planète, d'où une fréquentation croissante et privilégiée de ces deux cours.

\section{muryel.derlon@uibk.ac.at martina.mayer@uibk.ac.at}

\section{Muryel Derlon est traductrice-réviseuse depuis 1980,} spécialisée dans la traduction de textes médicaux, techniques et culturels (allemand $>$ français). Elle a travaillé pour des entreprises internationales et des musées de renom. Enseignante depuis 1985, elle amène ses étudiants à conduire des projets de traduction réels en équipe. Chevalier de l'Ordre des Palmes académiques, elle a reçu le prix Lehreplus décerné par l'université d'Innsbruck pour récompenser les enseignants s'étant distingués dans des projets didactiques. 
Martina Mayer est traductrice diplômée (français/espagnol/ anglais $>$ allemand) et enseignante-chercheuse. Elle dispense des cours en traduction générale, spécialisée et littéraire. En France, elle collabore avec différentes institutions, dont I'ISIT et l'université de Poitiers où elle assure depuis 2018 une unité d'enseignement d'introduction à la traductologie (licence/LTMI) se composant de deux cours. Ses recherches se concentrent sur les langues de spécialité, I'histoire et la didactique de la traduction. Elle réalise actuellement trois projets universitaires de traduction dont I'un a été primé par l'université d'Innsbruck.

\section{SOURCES CITÉES}

Bundesministerium für Digitalisierung und Wirtschaftsstandort, Bundesgesetz über die Organisation der Universitäten und ihre Studien (Universitätsgesetz 2002 - UG) [Fassung vom 13.2.2020], Wien, Rechtsinformationssystem des Bundes, 2020, https://www.ris.bka.gv.at/GeltendeFassung.wxe?Abfrage=Bundesnormen\&Ges etzesnummer=20002128, consulté le 2/06/2020.

KRENZLER-BEHM Dinah, Authentische Aufträge in der Übersetzerausbildung. Ein Leitfaden für die Translationsdidaktik, Berlin, Frank \& Timme, Coll. TRANSÜD. Arbeiten zur Theorie und Praxis des Übersetzens und Dolmetschens 58, 2013.

MAYER Martina, «Das können wir nicht: Wir sind ja keine Übersetzer! » in ZYBATOW Lew et al. (dir.), Übersetzen und Dolmetschen: Berufsbilder. Arbeitsfelder, Ausbildung. Ein- und Ausblicke in ein sich wandelndes Berufsfeld der Zukunft. 70 Jahre Innsbrucker Institut für Translationswissenschaft, Frankfurt am Main (Allemagne), Peter Lang, Forum Translationswissenschaft 21, 2017, p. 229-249.

Universität Innsbruck, Gesamtfassung ab 01.10.2016. Curriculum für das Bachelorstudium Translationswissenschaft an der Philologisch-Kulturwissenschaftlichen Fakultät der Universität Innsbruck, Innsbruck (Autriche), Fakultätenservicestelle der Universität Innsbruck, 2016, https://www.uibk.ac.at/fakultaeten-servicestelle/pruefungsreferate/gesamtfassung/ba-translationswissenschaft_stand01.10.2016.pdf, 2016, consulté le 2/06/2020.

Universität Innsbruck, Gesamtfassung ab 01.10.2019. Curriculum für das Masterstudium Translationswissenschaft an der Philologisch-Kulturwissenschaftlichen Fakultät der Universität Innsbruck, Innsbruck (Autriche), Fakultätenservicestelle der Universität Innsbruck, 2019, https://www.uibk.ac.at/fakultaeten-servicestelle/pruefungsreferate/gesamtfassung/ma-translationswissenschaft_ stand_01.10.2019.pdf, 2019, consulté le 2/06/2020. 